\title{
Acoustic Absorption with Material in Pieces and Back Air Layer
}

\author{
David Caballol ${ }^{1)}$, Álvaro P. Raposo ${ }^{2)}$ \\ 1) Department of Building Constructions and Control, Universidad Politécnica de Madrid, \\ Avda. Juan de Herrera, 6, 28040, Madrid, Spain. david.caballol@upm.es \\ 2) Department of Applied Mathematics, Universidad Politécnica de Madrid, \\ Avda. Juan de Herrera, 6, 28040, Madrid, Spain
}

\begin{abstract}
Summary
The sound absorption coefficient of three different materials is proved in a reverberation room against different layouts of the material and different back air layers, compared to those results where the same material is placed as a single piece. With the analysis of the data, a linear regression model is established in order to predict, for certain frequencies, the alteration produced in the absorption coefficient by using the same amount of material but placing it in patches separated from each other, instead of in one piece, and at different distances from the wall. The model is validated and proven to be robust and it is shown to be applicable to a variety of materials. It becomes a simple predictive tool which allows to estimate in situ the improvement in the absorption property of an absorbent material due to the separation of the patches.

PACS no. 43.55.Dt, 43.55.Ev, 43.55.Nd
\end{abstract}

\section{Introduction}

The extra absorption of a sound absorbent material when it is placed in patches rather than in one piece has been considered since long ago $[1,2,3,4]$ as well as recently $[5,6]$. This effect has been attributed to lack of diffusion and to the so called edge effect [7, 8]. Likewise, some research studies $[9,10]$ quantify the diffusion based on the scattering and absorption coefficient of the walls. Other researchers $[11,12,13,14]$ have studied how the edge effect increases the measured absorption in a reverberation chamber due to the extra surface area that is present because of the thickness of the sample under test. Yet other studies concentrate on absorbers based on the passive destructive interference principle (PDI) [15] or based on metamaterials $[16,17]$. However, none of these studies has found a simple method that allows to predict the improvement produced in the sound absorption of a dwelling, using a given amount of absorbent material but placing it in separated pieces. This is the goal of this paper: to find a simple method that allows an in situ estimation of the improved response of an absorbent material upon being placed in separated patches in a building work, instead of the usual theoretical models with many parameters [18] which are more difficult to apply in situ.

In Section 2 the method and materials utilized to obtain the data in the reverberation chamber are described in detail. In Section 3 data are dealt with by means of a variety of statistical tools. The result is a simple model of the dependence of the absorption coefficient on the variables occupation and air layer thickness. Finally, in Section 4 all the results are summed up in a sequence of brief conclusions.

\section{Materials and method}

The ISO 354 standard [19] for the measurement of the absorption coefficient of an absorbent material has guided the experimental procedure. The same method is commonly used in research studies for the characterization of construction materials $[20,21]$, though there are other available methods $[22,23]$. The method described in this standard measures the mean reverberation time in the reverberation room, for a given frequency, with and without the test sample. The sound absorption coefficient is calculated from these reverberation time periods through the equation

$$
\alpha_{S}=\frac{A_{T}}{S},
$$

where $S$ is the area of the surface of the sample of absorbent material, while $A_{T}=A-A_{0}$ stands for the equivalent absorbent area and is obtained as the difference between the absorbent area of the chamber with absorbent material, $A$,

$$
A=\frac{55.3 \mathrm{~V}}{c T}-4 \mathrm{Vm},
$$

and the absorbent area of the empty chamber, $A_{0}$,

$$
A_{0}=\frac{55.3 \mathrm{~V}}{c T_{0}}-4 V m_{0} \text {, }
$$


where $V$ is the volume of the chamber, in cubic metres, $c$ is the speed of sound, in meters per second, under the conditions of temperature and humidity of the experiment, $T$ and $T_{0}$ are, respectively, the reverberation time of the chamber, in seconds, with and without the absorbent sample and, finally, $m$ and $m_{0}$, are the power attenuation coefficients of air in the chamber when the data, with and without sample, where taken. In our case, the conditions of the experiment did not vary so the terms $m$ and $m_{0}$ cancel out and we get as the final expression for the absorption coefficient in terms of the measured reverberation times the following:

$$
\alpha_{S}=\frac{55.3 V}{c S}\left(\frac{1}{T}-\frac{1}{T_{0}}\right),
$$

which is the one we use in our analysis.

The testing conditions prescribe a specific reverberation room size and shape, with controlled temperature and humidity. The test sample must have an area $S$ between $10 \mathrm{~m}^{2}$ and $12 \mathrm{~m}^{2}$ and must be rectangular in shape with a width-to-length ratio between 0.7 and 1 .

The reverberation time has been measured in third octave bands with three different materials of similar thickness $(3 \mathrm{~cm}$, a thickness that is usually found in work sites for this type of materials), with different layouts of the material pieces and different thickness of the back air layer. The materials have been chosen on the basis of the following criteria: First, being regularly utilized in construction works. Second, having sufficiently different absorption properties. Therefore, two fibrous materials have been chosen, one of them with low density $\left(30 \mathrm{~kg} / \mathrm{m}^{3}\right)$ and another with high density $\left(100 \mathrm{~kg} / \mathrm{m}^{3}\right)$ and one porous material with very low density $\left(10 \mathrm{~kg} / \mathrm{m}^{3}\right)$, so that different flow resistivity values could be guaranteed [24]. The tested materials are the following:

- Material 1 (M1): non-woven polyester fibre $30 \mathrm{~mm}$ thick, in rigid planks with dimensions $1000 \mathrm{~mm} \times$ $500 \mathrm{~mm}$ and a density of $30 \mathrm{~kg} / \mathrm{m}^{3}$.

- Material 2 (M2): rock wool $30 \mathrm{~mm}$ thick, in rigid planks with dimensions $1000 \mathrm{~mm} \times 600 \mathrm{~mm}$ and a density of $100 \mathrm{~kg} / \mathrm{m}^{3}$.

- Material 3 (M3): melamine foam $30 \mathrm{~mm}$ thick, in rigid planks with dimensions $1000 \mathrm{~mm} \times 500 \mathrm{~mm}$ and a density of $10 \mathrm{~kg} / \mathrm{m}^{3}$.

For each material, the reverberation time was measured with three different thickness of the back air layer $(0 \mathrm{~cm}$, $5 \mathrm{~cm}$ and $15 \mathrm{~cm}$ ) and five layouts of the planks of the absorbent material. The samples have a net area of $10 \mathrm{~m}^{2}$ (in one piece), but the gross area has been increased through the separation of the planks in the different positions tested. As the patches are separated, the ratio between the net area of the patches (i.e. $10 \mathrm{~m}^{2}$ ) and the gross area they occupy diminishes from 1 (all patches together in a single piece) to $0.86,0.75,0.51$ and 0.37 . This ratio of net to gross area is the variable that is referred to as $O$, occupation, throughout the paper. Thus, each material has been tested in 15 different arrangements.

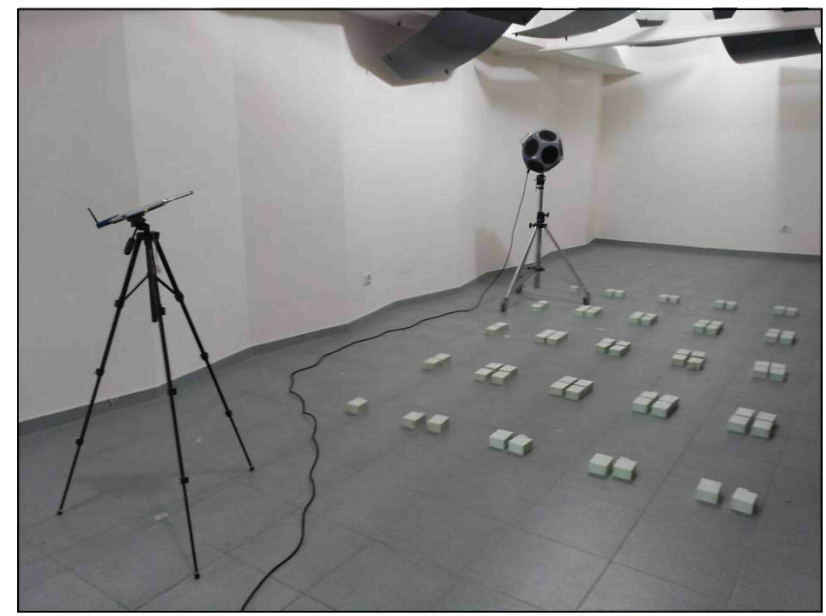

Figure 1. Empty reverberation chamber, with separators of $5 \mathrm{~cm}$.

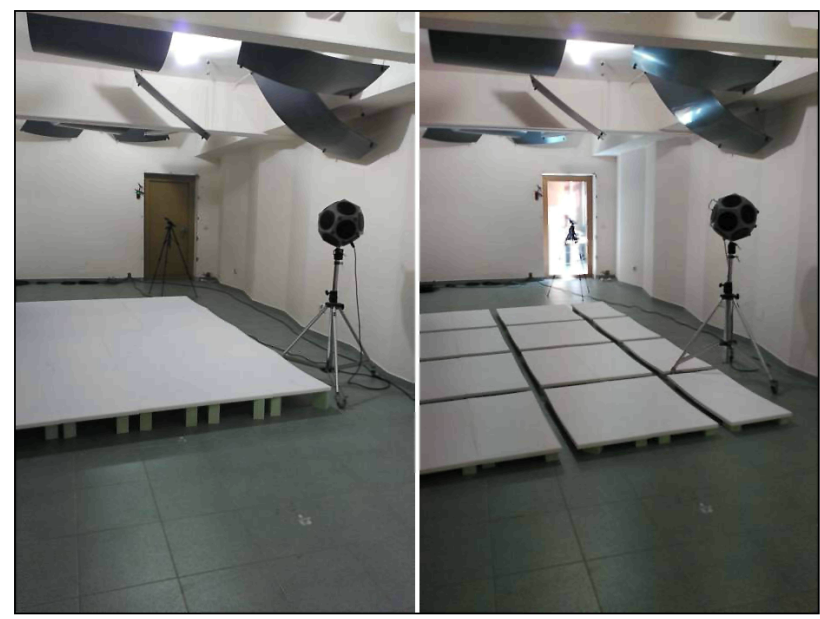

Figure 2. Two different arrangements of material M1: in the left side the material is laid in one piece (occupation 1) and with an air layer thickness of $15 \mathrm{~cm}$; in the right side the material is laid in separated pieces, with an occupation of 0.86 and separated $5 \mathrm{~cm}$ from the floor.

As was explained previously, we are searching for a simple in situ prediction method useful for technicians on an on site work or in a designer's office. Since the separation of the absorbent material in patches do not usually involve the covering of the sides of the patches, we have not covered them, our aim being to stay close to the real application procedures.

The separators are made of extruded polystyrene foam, a material without any acoustic absorption capabilities. For reference, the reverberation time in the empty chamber with the separators in their positions was also measured (see Figure 1).

The test samples are initially rectangular in shape, with a width/length ratio of 0.7 and are placed in such a way that every part is more than $1 \mathrm{~m}$ away from the edges of the reverberation room. This condition varies as the separation distance between the pieces increases, nevertheless a separation of at least $0.75 \mathrm{~m}$ was maintained (see Figure 2). 
In all cases, test sample pieces were allowed to reach a balance with the temperature and the relative humidity of the reverberation room before the tests were performed. The relative humidity of the chamber ranged from $38 \%$ to $39 \%$ during the tests, and the temperature between 19.9 and 20.6 degrees Celsius, so we took as the speed of sound for our computations $c=343.15 \mathrm{~m} / \mathrm{s}$ to be inserted in Equation (4). The volume of the chamber is $V=206 \mathrm{~m}^{3}$.

The interrupted noise signal method was used for measuring the reverberation time and the sound decay curves were measured from equivalent levels (using linear average) with integration times that vary between 20 milliseconds for the third octave bands of frequency 100 , 125 and $160 \mathrm{~Hz}$ and 10 milliseconds for the rest of frequency bands. Readings were made in all cases in third octave bands, as specified in the ISO 266 standard [25]. The standard ISO 354 [19] specifies a minimum of two source positions, of three receivers positions and a minimum of twelve combinations. Nevertheless, in our tests we have made eighteen measurements for each arrangement of each material, corresponding to six positions of the microphone and three positions of the noise source in the chamber.

In fact, each data is made as an average of three measurements taken with the same arrangement of material, noise source and microphone. It is known that three such measures may not be sufficient at low frequencies to gain a statistically significant average, but at this point we are bound by the experimental procedure chosen. In section 3 this point is further discussed once the data are analysed.

In specific occasions, we find a result of the reverberation time to be clearly wrong, and we decided to recalculate it on the basis of an improved estimation of the sound decay curve. In those cases we have changed slightly the standard evaluation range of $20 \mathrm{~dB}$ to a different range so as to improve the estimation. The question is to objectively detect those wrong estimations. To that end, the eighteen data are compared among them and a statistical criterion to detect outliers is applied to discard wrong estimations. The criterion chosen is the definition of extreme outliers, which is an adaptation of the original Tukey's criterion for detecting outliers in a boxplot [26]. The criterion is to rule out points outside the range $\left[Q_{1}-3 R, Q_{3}+3 R\right]$, where $Q_{1}$ and $Q_{3}$ are the first and third quartiles respectively and $R=Q_{3}-Q_{1}$ is the interquartile range of the eighteen data measured for each arrangement of the material and each frequency (Tukey's criterion uses 1.5 times $R$ below or above the quartiles). The points ruled out were calculated again. Once the data were debugged the mean of the eighteen points of each arrangement, each material and each frequency was taken, and the resulting data were fed to the statistical tools described in next section.

\section{Data analysis and results}

Once the data were debugged as explained in previous section, for each of the eighteen frequency bands we have

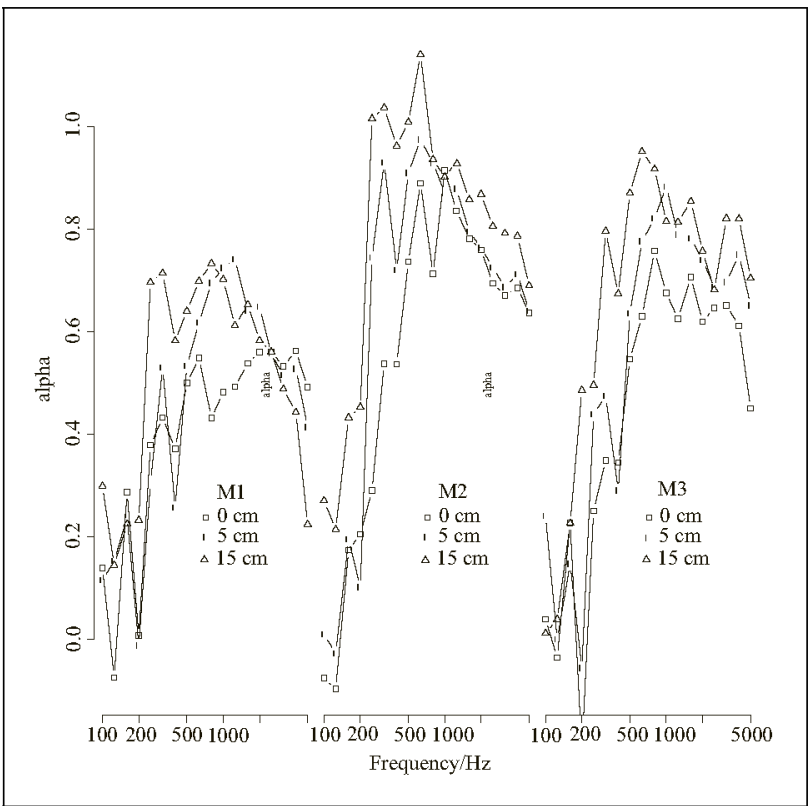

Figure 3. Absorption coefficient as a function of the frequency (in logarithmic scale) for the three different materials and the three thickness of the air layer, all of them for a fixed occupation of 1 , that is, all the patches in a single piece.

a complete factorial design with three factors: the material (with 3 levels, denoted M1, M2 and M3), the thickness of the back air layer (with 3 levels, $0 \mathrm{~cm}, 5 \mathrm{~cm}$ and $15 \mathrm{~cm}$ ) and, finally, the occupation variable (with $5 \mathrm{lev}$ els, $0.37,0.51,0.75,0.86$ and 1.00) which amounts to $3 \times 3 \times 5=45$ data. We also measured the reverberation time of the empty chamber in each frequency, so we were able to apply equation (4) and obtain 45 values of the absorption coefficient, which is our response variable under study. The explaining variables are the material, the thickness of the air layer in centimetres, denoted $\boldsymbol{B}$, and the occupation, denoted $O$. The goal is to quantify the effect of the variables $B$ and, mainly, $O$ on the absorption capabilities of the layout of a material by means of a model, as simple as possible, but which takes into account the possible interaction between the variables. In particular, we want to explore to what extent the dependence of the absorption properties on these variables is different for different materials.

To start with the analysis it is interesting to study some exploratory figures which naively show trends in the different variables.

In Figure 3 the role of the thickness of the air layer can be explored in terms of the frequency for the three materials, while the occupation is held fixed at $O=1$, that is, all the patches together in a single piece. The effect of the air layer is most noticeable at medium and high frequencies, the thicker the air layer, the higher the absorption coefficient. At low frequencies it is hard to distinguish a trend. This first observation has been widely discussed [27, pp. 185-186 ], [28, pp. 184-188]. However, we have to study if this visual conclusion is statistically significant, if it is consistent at other values of the occupation and if there 


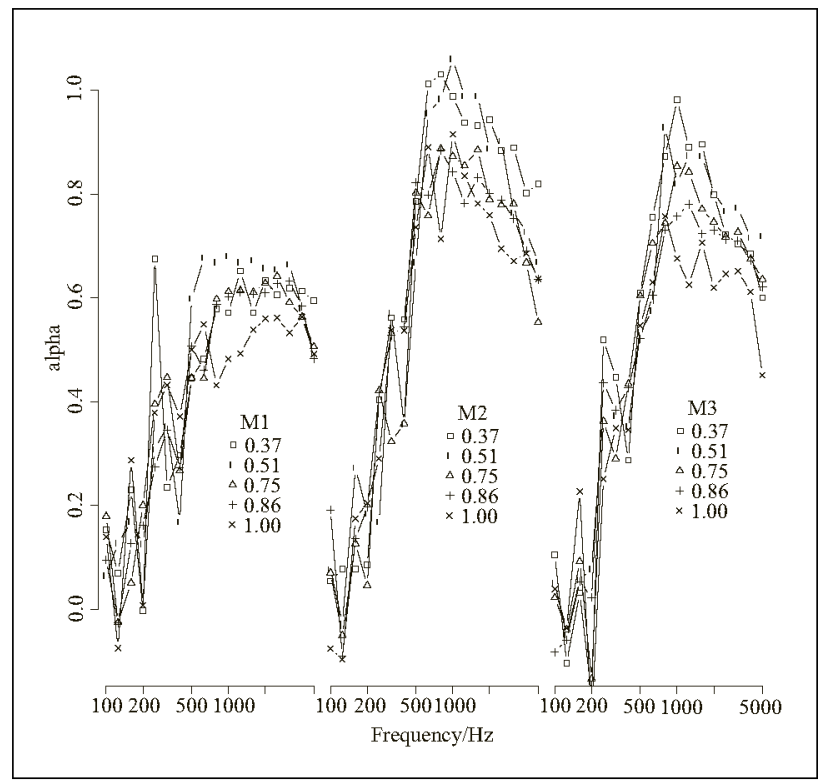

Figure 4. Absorption coefficient as a function of the frequency (in logarithmic scale) for the three different materials and the five levels of the occupation variable, all of them without back air layer.

is an interaction among these variables and the material. In Figure 4 the dependence on the variable occupation is shown for different materials at a fixed back air layer of $0 \mathrm{~cm}$ (i.e. without back air layer). In this case the figures are messy although, in general terms, we can distinguish slight trends at medium and high frequencies of higher absorption coefficient for lower occupation variable. Again, a statistical analysis must confirm or reject this visual inspection and make it extensible to other values of the air layer.

It is clear, thus, that a rigorous statistical analysis is needed in order to give significance to the naive observations, to extend them to all the cases of frequencies, materials, air layer thickness and levels of occupation and, if possible, to quantify the observed dependencies. In the following subsections a series of steps performs such a statistical analysis.

\subsection{First step: analysis of variance in each fre- quency}

An analysis of variance has been performed in each frequency, with 45 points, to see the relative importance of the variables (material, back air layer and occupation) in the explanation of the variability of the data. Table I shows the results of these analysis. Only the $p$-values of the $F$ test in each case are presented, including the main factors and interactions up to third order.

In the table the material and the air layer are seen to be the dominant factors in each frequency, except for the case of $100 \mathrm{~Hz}$, where no factor nor interaction seems to be significant. At the highest frequency of $5000 \mathrm{~Hz}$, the air layer is also a non significant factor in the explanation of the variability. This gives a wider role of the air layer than that suggested by Figure 3, where the air layer seems to affect mainly the medium range frequency bands. The effect of the occupation is the less important of the three, and is only significant starting from $800 \mathrm{~Hz}$ and above, until $4000 \mathrm{~Hz}$, in which it ceases to be a satisfactory explanatory variable of the reverberation time variability, which is approximately the same result stated by other authors [7]. Finally, the interactions among the three variables is negligible in most cases. The interaction between material and air layer is significant at low frequencies $(125$ to $250 \mathrm{~Hz})$ and also at high frequencies $(2000$ to $5000 \mathrm{~Hz})$. The interaction between material and occupation is only significant at $125 \mathrm{~Hz}$ and at high frequencies starting at $1600 \mathrm{~Hz}$ (except at $2500 \mathrm{~Hz}$ ). The interaction between the air layer and the occupation variables should only be taken into account at $125 \mathrm{~Hz}$ and $630 \mathrm{~Hz}$. Finally, there is no significant interaction of third order at any frequency.

Since the goal is to study the effect of the occupation variable by means of a simple model, the previous results bound us to consider only the medium frequencies, from $800 \mathrm{~Hz}$ to $4000 \mathrm{~Hz}$. However, the failure of the air layer and the occupation as explanatory variables at $5000 \mathrm{~Hz}$ deserves further study as the interaction of these variables with the material is statistically significant. Therefore, in the following sections we restrict ourselves to frequency bands from 800 to $5000 \mathrm{~Hz}$.

\subsection{Second step: a variety of models}

In this section a variety of models is proposed to explain the absorption coefficient as a function of the material, the air layer thickness and the occupation, for each frequency band in the range $800 \mathrm{~Hz}-5000 \mathrm{~Hz}$, and the best one is chosen among them. There are many models which can be fitted to the data, the more parameters the model, the better the fitting, but a model as simple as possible is always preferable. To that end four models have been fitted to the data and have been compared among them by means of the following $F$ test. Let $\mathrm{A}$ and $\mathrm{B}$ be two models with $p_{A}$ and $p_{B}$ parameters respectively, and assume that model $\mathrm{B}$ contains, for some combination of its parameters, model A. Therefore $p_{B}>p_{A}$ and the sum of squares of the residuals in model A must be greater than this sum in model $B$. The question to be elucidated is if the addition of more parameters to model A is worth of it. The statistic $[29,30]$

$$
F=\frac{n-p_{B}}{p_{B}-p_{A}} \frac{S_{A}-S_{B}}{S_{B}},
$$

where $S_{A}$ and $S_{B}$ are the sums of squares of the residuals of each model and $n$ is the size of the sample fitted with the models, has an $F$ distribution with $p_{B}-p_{A}$ and $n-p_{B}$ degrees of freedom under the null hypothesis that model B does not improve model $\mathrm{A}$. This test allows to compare the four models and hence accept or discard the more complex models with regard to the simpler ones.

All the models considered take into account the effect of the material, as a qualitative variable, the air layer thickness and the occupation, the latter two as continuous vari- 
Table I. Results of the analysis of variance on each frequency of the sample. The factors are the material, the air layer thickness and the occupation. For each one the $p$-values associated with the $F$ tests are shown for the main factors and the interactions up to third order.

\begin{tabular}{|cccccccc|}
\hline Freq. & material & air layer & occup. & mat-air & mat-occup & air-occup & mat-air-occup \\
\hline 100 & 0.1487 & 0.1743 & 0.1752 & 0.2737 & 0.9038 & 0.1559 & 0.2741 \\
125 & 0.0000 & 0.0000 & 0.1676 & 0.0046 & 0.0258 & 0.0005 & 0.1301 \\
160 & 0.0000 & 0.0003 & 0.0782 & 0.0687 & 0.1522 & 0.4944 & 0.1225 \\
200 & 0.0000 & 0.0000 & 0.4952 & 0.0079 & 0.7484 & 0.1277 & 0.4454 \\
250 & 0.0056 & 0.0000 & 0.9078 & 0.0290 & 0.1608 & 0.6093 & 0.5788 \\
315 & 0.0000 & 0.0000 & 0.4706 & 0.4107 & 0.9215 & 0.4569 & 0.2309 \\
400 & 0.0000 & 0.0000 & 0.7905 & 0.8822 & 0.8355 & 0.7180 & 0.6050 \\
500 & 0.0000 & 0.0000 & 0.9712 & 0.0813 & 0.6361 & 0.8634 & 0.1102 \\
630 & 0.0000 & 0.0000 & 0.1813 & 0.3798 & 0.9077 & 0.0144 & 0.8441 \\
800 & 0.0000 & 0.0000 & 0.0015 & 0.8415 & 0.4982 & 0.0900 & 0.8002 \\
1000 & 0.0000 & 0.0223 & 0.0015 & 0.9449 & 0.2350 & 0.1632 & 0.6293 \\
1250 & 0.0000 & 0.0101 & 0.0001 & 0.2676 & 0.4573 & 0.2907 & 0.8477 \\
1600 & 0.0000 & 0.0000 & 0.0000 & 0.9459 & 0.0101 & 0.1943 & 0.1477 \\
2000 & 0.0000 & 0.0000 & 0.0000 & 0.0352 & 0.0066 & 0.1171 & 0.6864 \\
2500 & 0.0000 & 0.0003 & 0.0001 & 0.0675 & 0.1234 & 0.8533 & 0.4346 \\
3150 & 0.0000 & 0.0028 & 0.0015 & 0.0011 & 0.0430 & 0.1230 & 0.8389 \\
4000 & 0.0000 & 0.0003 & 0.0031 & 0.0000 & 0.0008 & 0.5244 & 0.5091 \\
5000 & 0.0000 & 0.9577 & 0.1097 & 0.0000 & 0.0192 & 0.1868 & 0.2503 \\
\hline
\end{tabular}

ables. They differ in the interaction of these variables. The models are labeled $a, b, c$ and $d$.

Model $a$ is defined by the equation

$$
\alpha_{S}=\alpha_{i}+\beta O+\gamma B+e
$$

where $O$ and $B$ stand for the variables occupation and air layer respectively as stated previously, while $e$ is the stochastic term, which is assumed to be normally distributed with mean 0 and variance $\sigma^{2}$. The parameters of the model are $\alpha_{1}, \alpha_{2}, \alpha_{3}$, which are the effect of the materials M1, M2 and M3 respectively, $\beta$, the slope for the variable $O$, and $\gamma$, the slope for the variable $B$. The model has $p_{a}=5$ parameters to be estimated. Also the error variance, $\sigma^{2}$, has to be estimated. In this model the effect of each of the variables is collected separately, and no interaction among them is accounted for.

Model $b$ is defined by the equation

$$
\alpha_{S}=\alpha_{i}+\beta O+\gamma B+\delta O B+e,
$$

where the variables are the same. This model has a new parameter, $\delta$, which accounts for an interaction term between $B$ and $O$, thus making $p_{b}=6$ parameters. Model $b$ contains model $a$ by taking $\delta=0$.

Model $c$ is defined by the equation

$$
\alpha_{S}=\alpha_{i}+\beta_{i} O+\gamma_{i} B+e,
$$

where now the parameters are $\alpha_{i}, \beta_{i}$ and $\gamma_{i}, i=1,2,3$ for each material respectively, i.e., a model with a constant term, a slope for the variable $O$ and a slope for the variable $B$ for each material. This amounts to consider interaction among the material and the occupation and the material and air layer, but no interaction between occupation and air layer. The sum of squares of model $c$ is obtained by fitting the model to the 15 data of each material and then adding the sums of squares of the three models. The total number of parameters is thus $p_{c}=9$. This model contains model $a$ by taking $\beta_{1}=\beta_{2}=\beta_{3}$ and $\gamma_{1}=\gamma_{2}=\gamma_{3}$, but it does not contain model $b$, so they cannot be compared.

Finally, model $d$ is defined as

$$
T=\alpha_{i}+\beta_{i} O+\gamma_{i} B+\delta_{i} O B+e,
$$

where three more parameters are considered, $\delta_{i}, i=1,2,3$, which account for the possible interaction of the variables $\boldsymbol{B}$ and $\boldsymbol{O}$ and the material. The number of parameters is $p_{d}=12$ and it contains all the previous models by similar conditions on its parameters as pointed previously.

The four models have been fitted to the $n=45$ data in each frequency and then have been compared by means of the $F$ test aforementioned. The possible comparisons are $a-b, a-c, a-d, b-d$ and $c-d$. In Table II the values of the sums of squares of each model, and the $p$-value associated with the $F$ tests are gathered.

Looking at the column of the $p$-value of the comparisons between models $a$ and $b$ one discovers that the introduction of an interaction term between occupation and air layer is not worth the extra parameter, so model $a$ is preferable to model $b$. The same is deduced from the last column of the table, where models $c$ and $d$ are compared; in no case the addition of interaction parameters between occupation and air layer are worth of it. These two conclusions are consistent with the interpretation of Table I. Therefore, since models $a$ and $c$ are not improved by $b$ and $d$ respectively, the only comparison which is relevant is that of $a$ with $c$, that is the addition to model $a$ of interactions of the material with the occupation and with the air layer respectively. The $a-c$ column of the table says that model $c$ is slightly better than model $a$ for $2000 \mathrm{~Hz}$, and clearly better for 3150,4000 and $5000 \mathrm{~Hz}$, which is a confirmation of the results of Table I. 
Table II. Results of the $F$ test for the comparison of the four models in each frequency according to the statistic defined in Equation (5). The sums of squares of the residuals of each model are shown, as well as the $p$-value of the $F$ test of each comparison.

\begin{tabular}{|cccccccccc|}
\hline & \multicolumn{4}{c}{ s.s. of residuals } & \multicolumn{5}{c|}{$p$-values of the $F$ tests } \\
Freq. & $S_{a}$ & $S_{b}$ & $S_{c}$ & $S_{d}$ & a-b & a-c & a-d & b-d & c-d \\
\hline 800 & 0.31 & 0.28 & 0.29 & 0.27 & 0.0737 & 0.7813 & 0.6298 & 0.8928 & 0.3372 \\
1000 & 0.32 & 0.30 & 0.29 & 0.27 & 0.1516 & 0.5420 & 0.5375 & 0.6667 & 0.4090 \\
1250 & 0.23 & 0.22 & 0.20 & 0.19 & 0.2814 & 0.3558 & 0.5677 & 0.5917 & 0.6882 \\
1600 & 0.10 & 0.10 & 0.08 & 0.07 & 0.2385 & 0.0608 & 0.0452 & 0.0445 & 0.1428 \\
2000 & 0.06 & 0.06 & 0.04 & 0.04 & 0.1751 & 0.0035 & 0.0104 & 0.0114 & 0.3563 \\
2500 & 0.09 & 0.09 & 0.07 & 0.07 & 0.8632 & 0.0475 & 0.1376 & 0.0931 & 0.6314 \\
3150 & 0.11 & 0.10 & 0.07 & 0.06 & 0.1986 & 0.0009 & 0.0039 & 0.0039 & 0.4264 \\
4000 & 0.18 & 0.18 & 0.05 & 0.05 & 0.7200 & 0.0000 & 0.0000 & 0.0000 & 0.6212 \\
5000 & 0.46 & 0.45 & 0.17 & 0.15 & 0.4030 & 0.0000 & 0.0000 & 0.0000 & 0.2153 \\
\hline
\end{tabular}

Table III. Fitted parameters of model $a$, as given by Equation (6). Each parameter is given with its standard error. In addition the standard error of the residuals, $s_{R}$, is the estimation of $\sigma$. Also the $R^{2}$ and $p$-value of each fitting is given.

\begin{tabular}{|cccccccccccccc|}
\hline Freq. & $\alpha_{1}$ & $s_{\alpha_{1}}$ & $\alpha_{2}$ & $s_{\alpha_{2}}$ & $\alpha_{3}$ & $s_{\alpha_{3}}$ & $\beta$ & $s_{\beta}$ & $\gamma$ & $s_{\gamma}$ & $s_{R}$ & $R^{2}$ & $p$-value \\
\hline 800 & 0.77 & 0.05 & 1.09 & 0.03 & 0.99 & 0.03 & -0.20 & 0.06 & 0.010 & 0.002 & 0.09 & 0.78 & 0.0000 \\
1000 & 0.80 & 0.05 & 1.12 & 0.03 & 1.00 & 0.03 & -0.20 & 0.06 & 0.005 & 0.002 & 0.09 & 0.75 & 0.0000 \\
1250 & 0.79 & 0.04 & 1.07 & 0.03 & 1.01 & 0.03 & -0.22 & 0.05 & 0.005 & 0.002 & 0.08 & 0.78 & 0.0000 \\
1600 & 0.75 & 0.03 & 1.02 & 0.02 & 0.93 & 0.02 & -0.18 & 0.03 & 0.007 & 0.001 & 0.05 & 0.88 & 0.0000 \\
2000 & 0.72 & 0.02 & 0.97 & 0.01 & 0.86 & 0.01 & -0.17 & 0.03 & 0.005 & 0.001 & 0.04 & 0.90 & 0.0000 \\
2500 & 0.66 & 0.03 & 0.90 & 0.02 & 0.80 & 0.02 & -0.13 & 0.03 & 0.004 & 0.001 & 0.05 & 0.85 & 0.0000 \\
3150 & 0.60 & 0.03 & 0.85 & 0.02 & 0.80 & 0.02 & -0.10 & 0.03 & 0.003 & 0.001 & 0.05 & 0.83 & 0.0000 \\
4000 & 0.54 & 0.04 & 0.83 & 0.02 & 0.78 & 0.02 & -0.08 & 0.04 & 0.004 & 0.002 & 0.07 & 0.81 & 0.0000 \\
5000 & 0.42 & 0.06 & 0.77 & 0.04 & 0.72 & 0.04 & -0.07 & 0.07 & 0.000 & 0.003 & 0.11 & 0.70 & 0.0000 \\
\hline
\end{tabular}

\subsection{Fourth step: description of the model chosen and validation}

In this section models $a$ and $c$, which best explain the dependence of the absorption coefficient on the material, the occupation and the air layer thickness, are completely described. Their application is also discussed and their diagnostics is presented to validate them in each frequency.

Table III gives the parameters of model $a$, together with their standard errors, the standard error of the residuals, which is an estimation of $\sigma$ (the standard deviation of the stochastic term in Equation (6)), the computed $R^{2}$ and the $p$-value of the test of the fitting of the model. The fitted models at 3150,4000 and $5000 \mathrm{~Hz}$ are also shown since it helps to understand the situation in this regime of high frequencies.

In the table it is noticeable the high values of the computed $R^{2}$ which indicates that the model explain a great part of the variability observed in the data, around $80 \%$. Also the $p$-values are noticeable, all of them zero up to four decimal places, which tells us that in all cases the contribution of the explanatory variables is significant. However, the latter may be possibly accounted for only because of the material. In fact, for the three highest frequencies the slopes of the occupation and the air layer are very small becoming compatible with zero in the case of $5000 \mathrm{~Hz}$ in both variables, which means no dependence at all on these variables. For the other frequencies, 800 to $2500 \mathrm{~Hz}$, the slopes of the occupation as well as the air layer are sound numbers with a standard error which is an admissible figure. Notice the sign of each slope: the parameter $\beta$ is negative, hence the absorption coefficient is increased as the occupation is reduced, that is, the patches are separated. On the contrary, the parameter $\gamma$ is positive, showing that the thicker the air layer, the higher the absorption coefficient, as Figure 3 suggested.

Model $a$ must be validated against the hypothesis of model fitting prior to assume it correct. This is done in Figures 5 and 6.

In Figure 5 the residuals appear randomly distributed above and below the zero and no significant trends are visible, therefore supporting the hypothesis of linear correlation of the variables and independence. At 3150, 4000 and $5000 \mathrm{~Hz}$ the residues show a splitting into two groups, which are defined by the material as it is discussed below, but this splitting does not invalidate the previous conclusions. Also the hypothesis of homoscedasticity is confirmed by these graphs, as the dispersions of the residuals look similar at all points. Figure 6 confirms the hypothesis of normality of the residuals, as all the quantile-quantile plots show the points sufficiently aligned along the theoretical line of quantiles.

Table IV shows the parameters of model $c$ fitted to the data only for the high frequencies: 3150,4000 and $5000 \mathrm{~Hz}$.

The fitted parameters of model $c$ shown in Table IV deserve a brief discussion. The main difference between 


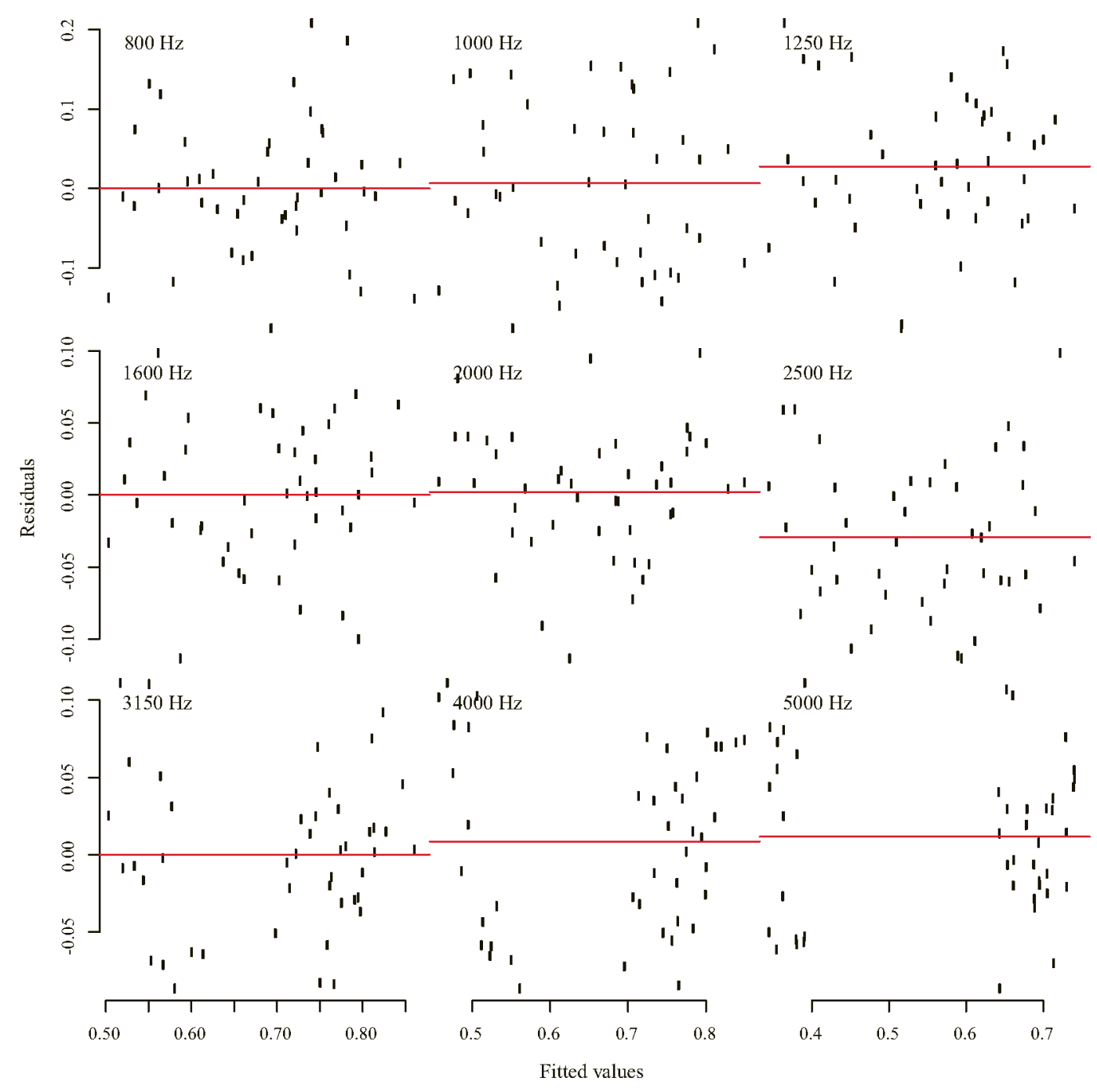

Figure 5. Residuals of model $a$ versus fitted values for each frequency. The horizontal line sets the zero in each graph.

Table IV. Fitted parameters of model $c$, as given by Equation (8), for the high frequencies 3150,4000 and $5000 \mathrm{~Hz}$. Each parameter is given with its standard error. In addition the standard error of the residuals, $s_{R}$, is the estimation of $\sigma$. Also the $R^{2}$ and $p$-value of each fitting is given.

\begin{tabular}{|ccccccccccc|}
\hline Freq. & Material & $\alpha$ & $s_{\alpha}$ & $\beta$ & $s_{\beta}$ & $\gamma$ & $s_{\gamma}$ & $s_{R}$ & $R^{2}$ & $p$-value \\
\hline \multirow{3}{*}{3150} & M1 & 0.61 & 0.05 & -0.04 & 0.06 & -0.003 & 0.002 & 0.05 & 0.13 & 0.4221 \\
& M2 & 0.90 & 0.04 & -0.20 & 0.05 & 0.006 & 0.002 & 0.04 & 0.74 & 0.0003 \\
& M3 & 0.74 & 0.03 & -0.05 & 0.03 & 0.006 & 0.001 & 0.03 & 0.70 & 0.0008 \\
& M1 & 0.55 & 0.03 & 0.00 & 0.04 & -0.007 & 0.002 & 0.04 & 0.62 & 0.0028 \\
5000 & M2 & 0.89 & 0.03 & -0.22 & 0.04 & 0.010 & 0.001 & 0.03 & 0.88 & 0.0000 \\
& M3 & 0.70 & 0.03 & -0.01 & 0.04 & 0.008 & 0.002 & 0.04 & 0.69 & 0.0009 \\
& M1 & 0.45 & 0.06 & 0.05 & 0.08 & -0.017 & 0.003 & 0.07 & 0.73 & 0.0004 \\
& M2 & 0.85 & 0.04 & -0.25 & 0.06 & 0.007 & 0.002 & 0.05 & 0.71 & 0.0006 \\
& M3 & 0.61 & 0.07 & -0.01 & 0.09 & 0.010 & 0.003 & 0.08 & 0.41 & 0.0418 \\
\hline
\end{tabular}

models $a$ and $c$ is that the latter accounts for an interaction of the material with the variables $O$ and $B$, and this is confirmed by the very different values of $\beta$ and $\gamma$ within a given third octave band but for different materials. In two cases, M1 at $3150 \mathrm{~Hz}$ and M3 at $5000 \mathrm{~Hz}$, the fitted model cannot be assumed right because the low value of $R^{2}$ or a high $p$-value. In addition, the values of the coefficient $\beta$, which accounts for the effect of occupation, is compati- 


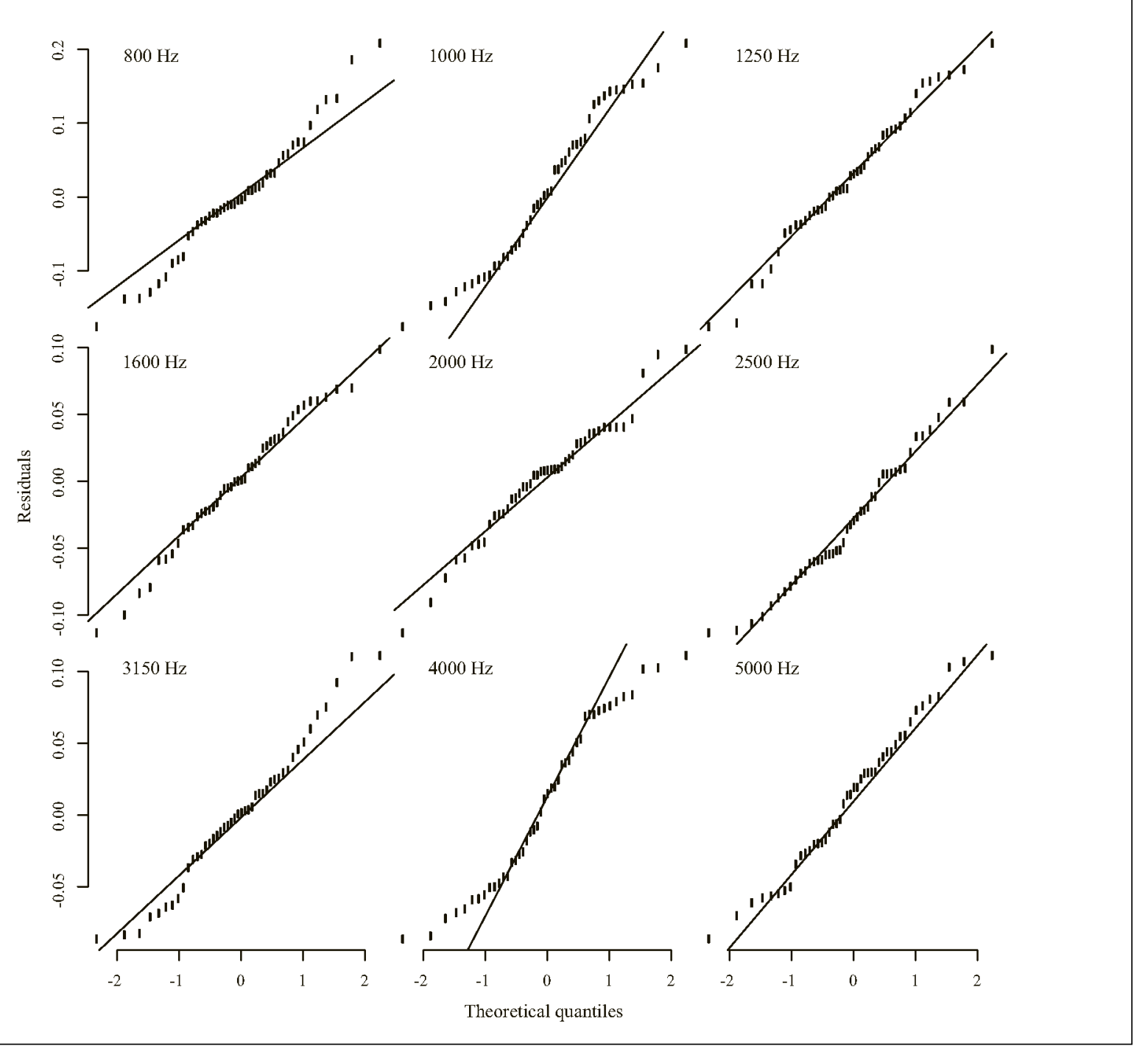

Figure 6. Quantile-quantile plot of the residuals of model $a$ under the assumption of normal distribution.

ble with zero in most cases within materials M1 and M3, which tells us that there is no significant effect of the variable occupation in the reverberation time. On the contrary, for material M2 the fitted models are all of them reliable, with high values of $R^{2}$ and low in the case of the $p$-value, and they all show nonzero values of the parameters.

Figures 7 and 8 show the diagnostics of model $c$ in the same way as it is done for model $a$ in Figures 5 and 6. It is noticeable that materials M1 and M3 present residuals plot with splitting of the points into three groups, which are related to the three values of the air layer thickness, and which show considerable deviations from the zero line in each group for 4000 and $5000 \mathrm{~Hz}$. Also the quantilequantile plots show non-negligible deviations from normality of the residuals in these cases.

Our conclusion is that model $c$, which is highly dependent on the material, gives a reliable result for material M2 but very poor results for M1 and M3. Since our aim is to obtain conclusions independent of the material, we are obliged to abandon model $c$ as suitable for high frequencies.

\subsection{Results independent of the material}

To end up with the model description we want to emphasize the part of the model which is independent of the materials tested. Fortunately, model $a$ gives the slopes of the variables occupation and air layer thickness independent of the material, so we can consider the value of the absorption coefficient with $B=0$, that is, with no back air layer, and $O=1$, i.e. all the patches of the material together in a single piece. Let us name this time $\alpha_{S S}$, the second $S$ after standard. By Equation (6) in the deterministic part of the model we have $\alpha_{S S}=\alpha_{i}+\beta$, so we can solve for $\alpha_{i}$ in terms of $\alpha_{S S}$ and $\beta$. Therefore we can write the deterministic part of model $a$ in the following form:

$$
\alpha_{S}=\alpha_{S S}+\beta(O-1)+\gamma B
$$

Now the parameters $\beta$ and $\gamma$ are given by the fitted model, in Table III, which we have extracted again in Table V. The dependence on the material is collected in $\alpha_{S S}$ but, since this number is the absorption coefficient in the conditions described by the standard, it can be obtained from 


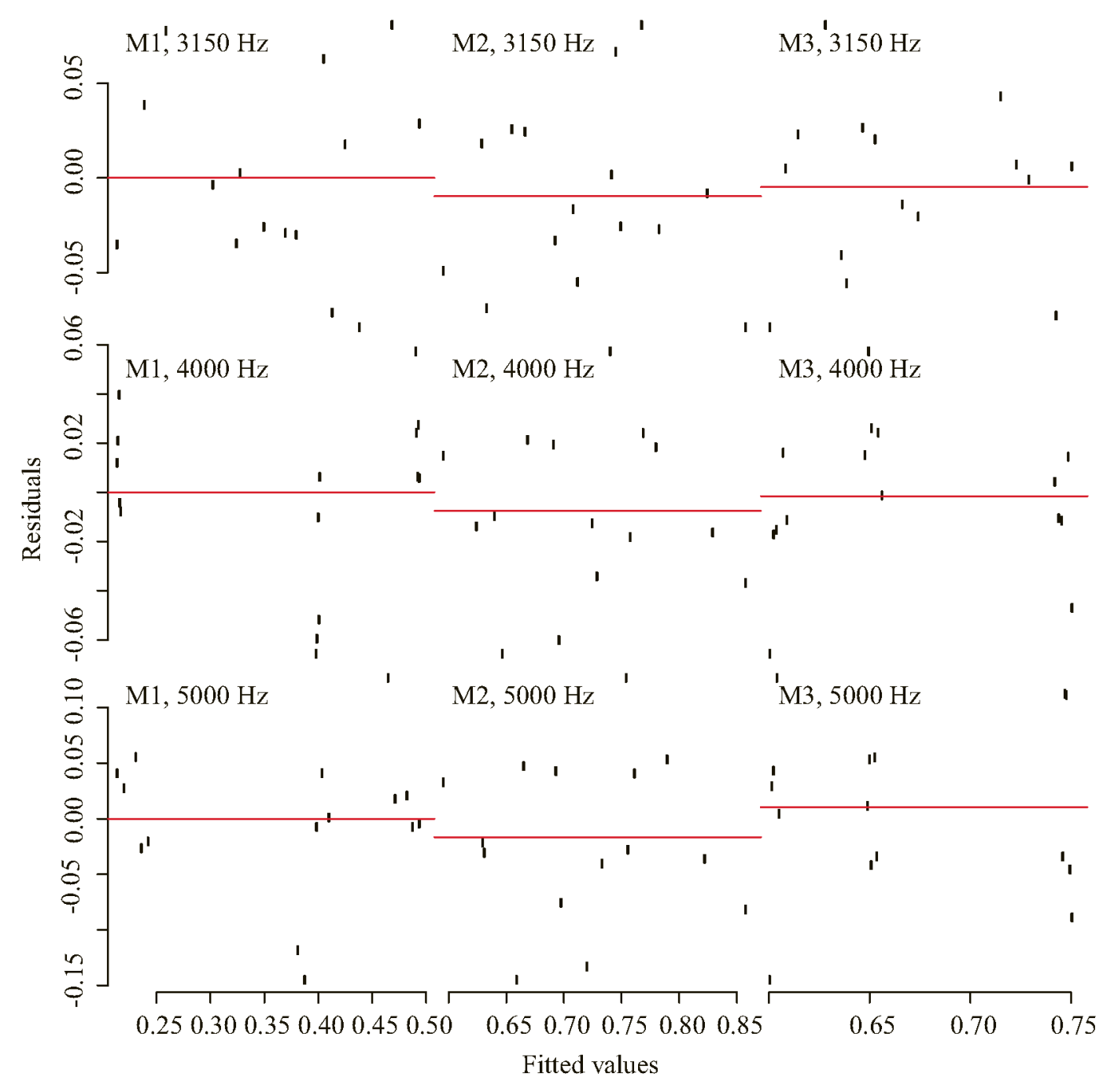

Figure 7. Residuals of model $c$ versus fitted values for each frequency and each material. The horizontal line sets the zero in each graph.

the technical specifications of the manufacturer. Equation (10) together with Table $\mathrm{V}$ is the main result of this paper. Equation (10) has been proved right from 800 to $5000 \mathrm{~Hz}$ but, as we have discussed previously, the highest frequencies should be described with model $c$. However, model $c$ for 3150,4000 and 5000 has proven wrong for certain materials and, in addition, the effect of the occupation and the air layer thickness are negligible, thus, we do not include them in this final summary.

\section{Conclusions}

The reverberation time is measured following the standard test in the reverberation room with three absorbent materials and with different arrangements both in the thickness of a back air layer and the splitting of the material in several separated pieces. After the measurement of the empty chamber reverberation time at each frequency, the absorption coefficients have been computed for each case. A statistically significant dependence has been found of
Table V. Slopes of the variables occupation $(\beta)$ and air layer thickness $(\gamma)$ of model $a$ with their respective standard error.

\begin{tabular}{|ccccc|}
\hline Freq. & $\beta$ & $s_{\beta}$ & $\gamma$ & $s_{\gamma}$ \\
\hline 800 & -0.20 & 0.06 & 0.010 & 0.002 \\
1000 & -0.20 & 0.06 & 0.005 & 0.002 \\
1250 & -0.22 & 0.05 & 0.005 & 0.002 \\
1600 & -0.18 & 0.03 & 0.007 & 0.001 \\
2000 & -0.17 & 0.03 & 0.005 & 0.001 \\
2500 & -0.13 & 0.03 & 0.004 & 0.001 \\
\hline
\end{tabular}

the absorption coefficient on the air layer thickness and the occupation variable in the range of 800 to $2500 \mathrm{~Hz}$. In this range a linear regression model has been successfully fitted to the data in each frequency, and the dependencies on the air layer thickness and the occupation have been found to be independent of the material under study. The output of the model is Equation (10) together with the parameters gathered in Table $\mathrm{V}$. The negative values of the 


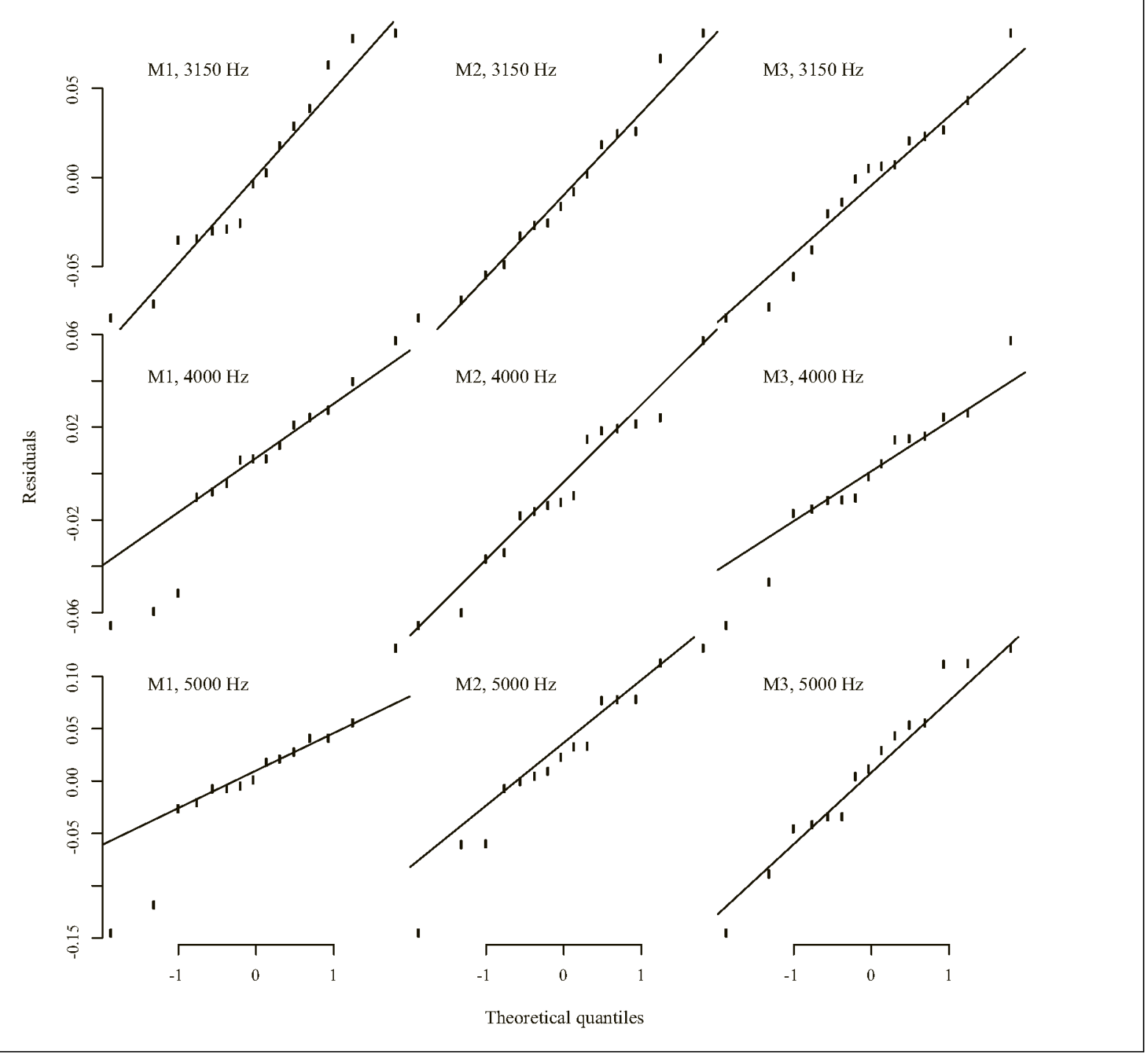

Figure 8. Quantile-quantile plot of the residuals of model $c$ under the assumption of normal distribution.

parameter $\beta$ give a quantitative information of the increase in the absorption coefficient as the patches of the material are placed separately. The positive values of the parameter $\gamma$ give the quantitative effect of increasing the absorption coefficient as the air layer thickness increases.

The linear model for each frequency in the range 800 to $2500 \mathrm{~Hz}$ has been validated in its departing hypothesis by the analysis of the residuals of the model, and the result in each case has been positive making all the models reliable.

For frequencies less than $800 \mathrm{~Hz}$ the occupation variable is not statistically significant in explaining the variability of data. This can be due to a higher dispersion of other factors, mainly the material or uncontrolled factors, or due to the need of more data at low frequencies as commented in page 557 . On the contrary, for higher frequencies, particularly 4000 and $5000 \mathrm{~Hz}$, there is a significant result on the role of the air layer thickness and the occupation: their slopes are compatible with zero, that is, the reverberation time is completely independent of them at these frequencies.
In this way, Equation (10) fulfills the purpose of enunciating a simple predictive model which allows, for absorbent materials in planks of $3 \mathrm{~cm}$ thickness, with the use of the coefficient of absorption of the material, to estimate in situ the improvement in its behaviour by placing it in separated pieces and with back air layer. But future work is needed to try to extend its applicability to lower frequencies.

\section{Acknowledgements}

Thanks are due to the staff and to the School of Engineering and Telecommunication Systems of the Universidad Politécnica de Madrid, as well as to the Department of Building Constructions and Control of the School of Building Engineering of Madrid. We are also grateful to two anonymous reviewers whose comments led to major changes in the original manuscript with the result of a much improved article. 


\section{References}

[1] V. Chrisler: Dependence of sound absorption upon the area and distribution of the absorbent material. J. Res. Natl. Bur. Stand. 13 (1934) 169-187.

[2] H. Feshbach, C. M. Harris: The effect of non-uniform wall distributions of absorbing material on the acoustics of rooms. J. Acoust. Soc. Am. 18 (1946) 472.

[3] R. Cook: Absorption of sound by patches of absorbent material. J. Acoust. Soc. Am. 29 (1957) 324-329.

[4] S. Thomasson: Theory and experiments of the sound absorption as function of the area. Dept. of Acoustics, Royal Institute of Technology, Stockholm, 1982, (Report TRITATAK8201).

[5] R. Lanoye, G. Vermeir, W. Lauriks, F. Sgard, W. Desmet: Prediction of the sound field above a patchwork of absorbing materials. J. Acoust. Soc. Am. 123 (2008) 793.

[6] J. Trevor, P. D'Antonio: Acoustics absorbers and diffusers. Taylor \& Francis, New York, 2009, Ch. Hybrid surfaces, 388-393.

[7] T. T. Wolde: Measurements on the edge-effect in reverberation rooms. Acustica 18 (1967) 207-212.

[8] H. Dekker: Edge effect measurements in a reverberation room. J. Sound Vib. 32 (1974) 199-202.

[9] A. Hanyu: Theoretical framework for quantitatively characterizing sound field diffusion based on scattering coefficient and absorption coefficient of walls. J. Acoust. Soc. Am. 128 (2010) 1140-1148.

[10] T. Cox, B. Dalenback, P. D'Antonio, J. Embrechts, J. Jeon, E. Mommertz, M. Vörlander: A tutorial on scattering and diffusion coefficients for room acoustic surfaces. Acta Acust. united Ac. 92 (2006) 1-15.

[11] A. Bruijin: Calculation of edge effect of sound absorbing structures. Dissertation. Delft, Holland, 1967.

[12] T. Bartel: Effect of absorber geometry on apparent absorption coefficients as measured in a reverberation chamber. J. Acoust. Soc. Am. 69 (1981) 1065-1074.

[13] D. Guicking: Theoretical evaluation of the edge effect of an absorbing strip of a pressure-release boundary. Acustica 70 (1990) 66-75.

[14] F. Kawakami: Deep-well approach for cancelling the edge effect in random incident absorption measurement. J. Acoust. Soc. Jpn. 19 (1998) 327-338.
[15] F. Setaki, M. Tenpierik, M. Turrin, A. van Timmeren: Acoustic absorbers by additive manufacturing. Build. Environ. 72 (2014) 188-200.

[16] Y. J. Kim, J. S. Hwang, Y. J. Yoo, B. X. Khuyen, X. Chen, Y. Lee: Triple-band metamaterial absorber based on single resonator. Curr. Appl. Phys. 17 (2017) 1260-1263.

[17] N. Dauchez, B. Nennig, O. Robin: Additional sound absorption within a poroelastic lamella network under oblique incidence. Acta Acust. united Ac. 104 (2018) 211-219.

[18] M. Ouisse, M. Ichchou, S. Chedly, C. M.: On the sensitivity analysis of porous material models. J. Sound Vib. 331 (2012) 5292-5308.

[19] ISO 354:2003: Acoustics - measurement of sound absorption in a reverberation room. Geneva, Switzerland, 2015.

[20] C. Díaz, M. Jiménez, M. Navacerrada, A. Pedrero: Acoustic properties of reed panels. Mater. Construcc. 62 (2012) $55-66$.

[21] J. Ramis, R. del Rey, J. Alba, L. Godinho, J. Carbajo: A model for acoustic absorbent materials derived from coconut fiber. Mater. Construcc. 64 (2014) e008.

[22] ISO 10534-2:1998: Acoustics-determination of sound absorption coefficient and impedance in impedance tubes-part 2: Transfer-function method. Geneva, Switzerland, 2002.

[23] E. Tijs, E. Druyvesteyn: An intensity method for measuring absorption properties in situ. Acta Acust. united Ac. 98 (2012) 342-353.

[24] D. A. Bies, C. H. Hansen: Flow resistance information for acoustical design. App. Acoust. 13 (1980) 357-391.

[25] ISO 266:1997: Acoustics - preferred frequencies. Geneva, Switzerland, 2013.

[26] J. W. Tukey: Exploratory data analysis. Addison-Wesley, Reading, MA, 1977.

[27] F. Everest, K. Pohlmann: Master handbook of acoustics. McGraw-Hill, New York, 2000.

[28] H. Kuttruff: Room acoustics. Spon press, New York, 2009, Ch. Reverberation and steady-state energy density.

[29] S. Weisberg: Applied linear regression. second ed. John Wiley \& Sons, New York, 1985.

[30] X. Yan, X. Gang Su: Linear regression analysis. World Scientific, Singapore, 2009.

[31] D. Caballol, A. P. Raposo: Acoustic absorption increase prediction by placing absorbent material in pieces. Appl. Acoust. 113 (2016) 185-192. 